\title{
R-Cadherin Is a Pax6-Regulated, Growth-Promoting Cue for Pioneer Axons
}

\author{
Gracie L. Andrews and Grant S. Mastick \\ Departments of Biology and Biochemistry, Cell and Molecular Biology Graduate Program, University of Nevada, Reno, Nevada 89557
}

The transcription factor Pax6 has been implicated in two processes that may be related in brain development: establishment of regional cell adhesion properties and axon guidance. In Pax6 mutant mouse embryos, forebrain pioneer axons make pathfinding errors. These errors occur in a region of the ventral thalamus in which the expression of the cell adhesion molecule R-cadherin (Cdh4) is lost in Pax6 mutants. In vitro, an R-cadherin substrate promoted pioneer axon outgrowth. Furthermore, pioneer axon outgrowth was rescued in vivo by selective replacement of R-cadherin by electroporation into cultured Pax6 mutant embryos. Thus, these studies implicate Pax6 as an early brain patterning gene that establishes regional adhesive codes to guide pioneer axons.

Key words: axon guidance; cadherin; transcription factor; pioneer axon; mutant; electroporation

\section{Introduction}

In early brain development, pioneer axons grow through the brain along specific trajectories, establishing the first organized tracts. The precise navigation by axons implies responsiveness to molecular cues in specific regions. Axons form two types of tracts: longitudinal (ascending or descending the CNS) or commissural (across the midline). For commissural axons, a dominant mechanism for guidance is chemoattraction (coupled with repulsion) to guide axons toward or away from the midline (Dickson, 2002). In contrast, for longitudinal axons, although constituting the majority of axons, navigation mechanisms are poorly defined. Local directional cues may be important, as suggested by surgical manipulations of substrate tissue (Hibbard, 1965; Harris, 1989; Nakamura et al., 2000). Additionally, repulsion from midline structures may keep axons coursing in longitudinal pathways (Kidd et al., 1998). As another mechanism of guidance, early pioneer axons establish the initial tracts, with later following axons guided by selective fasciculation onto the initial tracts (Chitnis and $\mathrm{Ku}$ wada, 1991; Anderson and Key, 1999; Treubert-Zimmermann et al., 2002). However, the molecular mechanisms that establish the initial longitudinal tracts remain mostly undefined.

One mechanism for axon navigation is selective adhesion between axons and their substrates. This type of adhesion can be mediated by cadherins. Cadherins are transmembrane cell adhesion molecules with three key features: a large number of subtypes, diverse spatially restricted expression patterns in the developing brain, and homophilic binding specificity (Redies, 2000).

Received June 16, 2003; revised Sept. 3, 2003; accepted Sept. 3, 2003.

This work was supported by National Institutes of Health Grants NS33337 (S. S. Easter, University of Michigan, Ann Arbor, MI) and HD38069 (G.S.M.), a Nevada Agricultural Experiment Station grant (University of Nevada, Reno), and startup funds from the University of Nevada, Reno. We thank M. Takeichi for R-cadherin antibody, G. Dressler for $\mathrm{Rcad}^{+}$cell lines, D. Egender, E. Hickey, and C. Corley-Mastick for cell culture advice, and N. Osumi and T. Inoue for expression plasmids and advice on whole embryo culture and electroporation.

Correspondence should be addressed to Grant Mastick, Department of Biology/200, University of Nevada, Reno, NV 89557. E-mail: gmastick@unr.edu.

Copyright $\odot 2003$ Society for Neuroscience $\quad$ 0270-6474/03/239873-08\$15.00/0
These features make cadherins compelling candidates for regulating the highly specific cellular interactions required for establishing neuronal connections in the brain. A number of observations support this idea. Functional circuits of neurons often share expression of specific cadherins (Redies et al., 1993; Liu et al., 1999). Furthermore, interference with axonal cadherins can inhibit axon outgrowth (Riehl et al., 1996; Stone and Sakaguchi, 1996), whereas forced expression of cadherins directs selective fasciculation with existing tracts (Treubert-Zimmermann et al., 2002). Importantly, cadherins are expressed in complex patterns in the developing brain. However, remaining undefined are the factors that establish specific cadherin patterns in the developing brain and the potential role of these patterns in guiding axons.

Mouse embryos mutant for the transcription factor Pax6 provide a testing ground for investigating axon guidance by cadherin-mediated adhesion. At embryonic day 10.5, (E10.5), the first longitudinal axons in the forebrain pioneer the tract of the postoptic commissure (TPOC) (Easter et al., 1993; Mastick and Easter, 1996). In Pax6 mutant embryos, the TPOC axons make severe pathfinding errors in the ventral thalamus (VT). These observations imply that important guidance cues within the TPOC pathway are regulated by Pax6. Interestingly, studies of older stages (E12.5-E18.5) suggested that R-cadherin (Rcad) mediates Pax6 function in cell adhesion: R-cadherin expression extensively overlaps with Pax6 (Matsunami and Takeichi, 1995), and R-cadherin expression is lost from VT of Pax6 mutants on E12.5 and later (Stoykova et al., 1997). Together, these observations suggest that Pax6 influences pioneer axons through the regulation of R-cadherin expression in VT. To directly test this hypothesis, we examined the relationship between migrating TPOC axons, Pax6, and R-cadherin.

\section{Materials and Methods}

Mice and embryo collection

Pax6 homozygous mutant embryos (and wild-type littermates as controls) were obtained from crosses between Pax6 heterozygous mice (the Sey-neu allele) in an FVB background, with day of vaginal plug desig- 
nated E0.5. In Pax6 heterozygous embryos, TPOC axons navigate identically to wild type (Mastick et al., 1997), so only homozygous mutant embryos were analyzed for this study. For each type of labeling experiment (antibody and in situ hybridization), three or more embryos of each genotype were analyzed. For embryo culture experiments (see below), homozygous mutant embryos could be recognized through the yolk sac by the altered morphology of the optic vesicle and nasal prominences. Mouse care and procedures were performed as approved by the University of Nevada, Reno Institutional Animal Care and Use Committee.

\section{In situ hybridization and antibody labeling}

Whole-mount in situ hybridization used digoxigenin-labeled antisense RNA probes, detected by anti-digoxigenin antibody coupled to alkaline phosphatase, according to the procedure of Wilkinson (1992), as modified (Parr et al., 1993). Pax6 probe made from a plasmid containing an EcoRI/NheI restriction fragment from mouse Pax6 cDNA was a gift of K. Backs and P. Gruss (Gottingen, Germany) (Walther and Gruss, 1991). $\mathrm{R}$-Cadherin probe was made from a plasmid containing a mouse R-cadherin cDNA fragment.

R-Cadherin antibody labeling used the MRCD5 rat monoclonal antibody, a gift from M. Takeichi (Kyoto University, Kyoto, Japan) (Matsunami and Takeichi, 1995). For R-cadherin antibody labeling, embryos were fixed in $4 \%$ paraformaldehyde for $1 \mathrm{hr}$, embedded in gelatin, and then frozen (Mastick and Andrews, 2001). After cryosectioning and placing on slides, gelatin was removed by incubation in warm $0.1 \mathrm{M}$ phosphate buffer, $\mathrm{pH}$ 7.4. Sections were fixed for $15 \mathrm{~min}$ on ice in $4 \%$ paraformaldehyde in HBSS (per liter, $2.38 \mathrm{gm}$ of HEPES, $8 \mathrm{gm}$ of $\mathrm{NaCl}, 0.4$ gm of $\mathrm{KCl}$, and $0.047 \mathrm{gm}$ of anhydrous $\mathrm{Na}_{2} \mathrm{HPO}$, with $\mathrm{pH}$ adjusted to 7.6 with $\mathrm{NaOH}$, and $\mathrm{CaCl}_{2}$ and $\mathrm{MgCl}_{2}$ added to final concentration of 1 $\mathrm{mM})$. After rinsing in HBSS, sections were permeabilized for $15 \mathrm{~min}$ with methanol at $-20^{\circ} \mathrm{C}$. To reconstitute immunoreactivity, the slides were then placed in a jar of HBSS and microwaved at high power for $14 \mathrm{sec}$ (just to the boiling point). The slides were then rinsed in TBS- $\mathrm{Ca}^{2+}$ buffer (per liter, $6.1 \mathrm{gm}$ of TrisHCl, $8.8 \mathrm{gm}$ of $\mathrm{NaCl}, 0.2 \mathrm{gm}$ of $\mathrm{CaCl}_{2}-$ $\mathrm{H}_{2} \mathrm{O}$, and $0.5 \%$ Triton $\mathrm{X}-100$, with $\mathrm{pH}$ adjusted to 7.6 with $\mathrm{HCl}$ ) and blocked for $30 \mathrm{~min}$ in TBSM (5\% dry skim milk in TBS-Ca ${ }^{2+}$ ). MRCD5 antibody supernatant was then incubated, undiluted, at room temperature overnight. After several rinses of TBSM for $5 \mathrm{~min}$ each, the slides were incubated overnight with biotin anti-rat secondary antibody (Jackson ImmunoResearch, West Grove, PA) diluted 1:200 in TBSM. After several rinses of TBSM for $5 \mathrm{~min}$ each, the slides were incubated in Cy2-avidin (Jackson ImmunoResearch) diluted 1:100 in TBSM for several hours. The slides were then labeled with Pax6 rabbit antisera, as described previously at 1:2000 dilution (Mastick et al., 1997) (antibody available from Chemicon, Temecula, CA), visualized with Cy2 antirabbit secondary antibody.

\section{Axon labeling}

Axons were traced in whole, paraformaldehyde-fixed embryos using DiI (Molecular Probes, Eugene, OR) as described previously (Mastick et al., 1997). For combining axon labels with in situ hybridization, DiI-labeled embryos were photoconverted under fluorescent light in the presence of diaminobenzidine (DAB) (Mastick et al., 1997).

\section{Axon outgrowth assay}

For substrate cells, mouse L fibroblast cells, which normally lack any cadherin expression (Matsunaga et al., 1988), or an L cell line stably transfected with R-cadherin (gift from G. Dressler, University of Michigan, Ann Arbor, MI) were plated onto multi-chamber tissue culture slides (Lab-Tek II chamber slides; Nalge Nunc International, Rochester, NY) and allowed to adhere at subconfluent density. Culture media was DMEM-F-12 media (Sigma, St. Louis, MO), supplemented with bicarbonate, penicillin-streptomycin, and $10 \%$ bovine calf serum (Harlan Sprague Dawley, Indianapolis, IN). The substrate cells were cultured to confluency. R-Cadherin expression in the $\mathrm{Rcad}^{+}$line was verified by immunofluorescence using MRCD5 antibody (see Fig. 3, insets). Explanted tissue containing TPOC neuron cell bodies was dissected from the area of the presumptive optic chiasm from E11.5 mouse embryos, cut into small pieces using microscalpels, and transferred by micropipette onto the culture substrate cells. After incubation for $24 \mathrm{hr}$, the slides with explants and substrate cells were fixed in $4 \%$ paraformaldehyde in $0.1 \mathrm{M}$ $\mathrm{PO}_{4}$ buffer.

To visualize the axons, the TuJ1 monoclonal antibody against neuronspecific $\beta$ III-tubulin (Babco, Richmond, CA) was used at 1:2000 dilution, as described previously (Easter et al., 1993; Mastick and Easter, 1996). The antibody labeling was visualized with Cy3 anti-mouse secondary antibody, and images were collected of each explant. To quantify axon outgrowth, the method of Matsunaga et al. (1988) was used on images of the explants to measure the area over which the axons extended. Because the area of each piece of explanted tissue varied and therefore contained varying numbers of neuron cell bodies, the area of the explanted tissue was subtracted from the total area.

\section{Electroporation and whole embryo culture}

\section{Embryo dissection and culture}

Early E10.5 (31-34 somites) embryos were prepared by standard dissection techniques for whole embryo culture (Osumi and Inoue, 2001). All dissections were done in Tyrodes's saline buffer, within $1 \mathrm{hr}$ of uterus collection. In brief, the decidual masses were dissected from uteri, and the decidual layers were removed, starting from the placental end. The placenta was left intact. The yolk sac was exposed by removal of Reichert's membrane, and mutant embryos were identified through the yolk sac by their morphology. A slit was made in the yolk sac by cutting between blood vessels. The anterior half of the embryo was pushed through this slit and exposed by opening of the amniotic membrane. The embryos were then placed into small roller bottles containing culture media (rat serum, collected by the immediate centrifugation method, and supplemented with $2 \mathrm{mg} / \mathrm{ml}$ glucose), and the air space was flushed with $60 \%$ oxygen, $35 \%$ nitrogen, and $5 \%$ carbon dioxide. The bottles were then placed on a rotator at $37^{\circ} \mathrm{C}$ for a $1 \mathrm{hr}$ incubation period preceding electroporation.

\section{Electroporation}

Each embryo was removed from the roller bottle using a transfer pipette and washed briefly in a Petri dish of Tyrode's saline. The embryo was then placed in a electroporation chamber with two platinum plates $(1.5 \mathrm{~cm}$ apart) in Tyrode's saline. A small hole was made in the roof of the hindbrain to relieve pressure caused by the injection. Approximately $0.2 \mathrm{ml}$ of plasmid DNA [2 $\mathrm{mg} / \mathrm{ml}$ pMiW-Rcad plus $1 \mathrm{mg} / \mathrm{ml}$ pCA-green fluorescent protein (GFP) in $\mathrm{PBS}$ ] was pressure injected into the forebrain ventricle using a fine micropipette inserted through the optic vesicle, with the flow directed against the VT/dorsal thalamus (DT) region. The embryo was then positioned so that the left side of the head was oriented toward the cathode. Square wave pulses (five pulses, $50 \mathrm{msec}, 60 \mathrm{~V}$ ) were delivered with a BTX (Holliston, MA) electroporator. The embryo was briefly washed in Tyrode's saline and returned to roller bottle culture for $12 \mathrm{hr}$.

\section{Expression plasmids}

The following plasmids, containing strong constituitive chick actin promoters, were used for electroporation. The R-cadherin expression plasmid was pMiW-Rcad (Matsunami et al., 1993); the cadherin-6 plasmid was pCA-Cad6 (Inoue et al., 2001); the GFP marker plasmid was pCAGFP (Inoue et al., 2001). The function of the R-cadherin expression plasmid was verified using R-cadherin antibody labeling of sections of electroporated embryos (data not shown).

\section{Assessment of electroporation targeting}

After the completion of culture, the embryos were fixed overnight at $4{ }^{\circ} \mathrm{C}$ in $4 \%$ paraformaldehyde in $0.1 \mathrm{~m}$ phosphate buffer, $\mathrm{pH}$ 7.4. Images were collected of the GFP pattern on the targeted side of each embryo (see Fig. $4 E)$. The VT/DT boundary was a visible landmark. Embryos that had strong fluorescence and coverage of the VT/DT region of the TPOC pathway were used for additional analysis.

\section{Axon analysis}

Axon growth in electroporated embryos was assessed by DiI labeling of the TPOC (Mastick et al., 1997). Small crystals of DiI were placed at the 

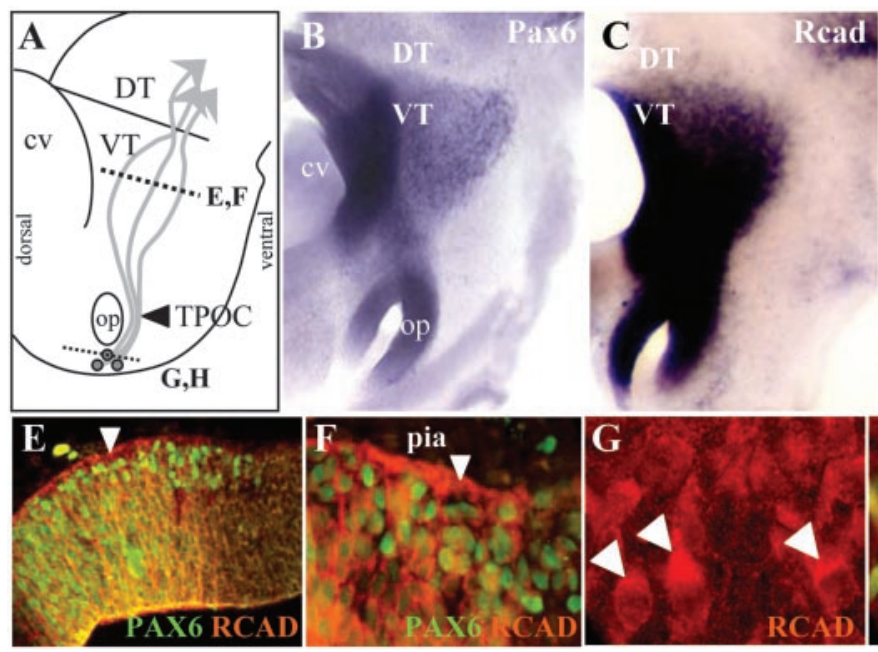

Figure 1. Pioneer axon growth through the Pax $6^{+} \mathrm{Rcad}^{+}$ventral thalamus. $A$, The pathway taken by TPOC axons through the embryonic mouse forebrain. The axons originate from a cluster of neurons located at the base of the optic stalk (op). The axons pass the optic stalk, continue through VT, and then cross into DT. Planes of section for $E-H$ are indicated by dashed lines. $B-D, E 10.5$ embryos labeled by whole-mount in situ hybridization (purple) for Pax6 $(B)$ or R-cadherin ( $C$ ), showing overlapping expression in VT.D, An embryo double labeled for R-cadherin (light purple) and TPOC axons (brown). The boundary of the Rcad ${ }^{+}$VT is indicated by the dashed line. E, F, Comparison of Pax6 and Rcad expression patterns by antibody labeling of sections. E, Section through VT, showing antibody labeling for Pax6 (green, nuclear) and Rcad (red, cell surface). A concentrated area of Rcad labeling is at the pial surface (arrowhead). F, High magnification showing the Rcad ${ }^{+}$layer at the pial surface (arrowhead). G, Section through TPOC cell bodies (arrowheads), labeled with Rcad antibody (red). $H$, Same section, with merged image of Rcad (red) and neuron-specific $\beta$-tubulin ( $\beta$ tub) antibody (green). Scale bar (in D): $A-D, 200 \mu \mathrm{m} ; E, 100 \mu \mathrm{m} ; F, 50 \mu \mathrm{m}$ in F; G, H, $25 \mu \mathrm{m}$.

ventral base of the optic stalk, bilaterally at the location of the TPOC neuron cell bodies. Embryos were then incubated for $2 \mathrm{~d}$ at $37^{\circ} \mathrm{C}$ in $4 \%$ paraformaldehyde in $0.1 \mathrm{M}$ phosphate buffer, $\mathrm{pH}$ 7.4. Fluorescent axon labeling was then examined with an epifluorescence microscope. If the tracts were bilaterally labeled, then the skin and mesenchyme overlying the forebrain was removed by dissection. Embryos were bisected and flat mounted on a depression slide, under a coverslip to partially flatten, thus facilitating counting the number of axons that crossed the VT/DT boundary. The axon numbers were an underestimate, especially for rescued TPOC outgrowth, because axon bundles were likely often counted as a single axon.

\section{Critical parameters for electroporation experiments}

The only embryos used for axon analysis were those that were at an optimal developmental stage (31-34 somites), grew well in culture, were effectively electroporated, and had strong bilateral DiI labeling of the TPOC axons.

Developmental staging. Optimal developmental stages were determined by analysis of TPOC axon growth in wild-type embryos. Staging of TPOC axon growth in wild type using DiI labeling showed that the first TPOC axons entered the VT region at 31 somites and crossed the VT/DT boundary by 34 somites (F. Nural and G. S. Mastick, unpublished observations). Therefore, Pax6 mutant embryos for electroporation were selected from litters with embryos with somite numbers ranging from 31 to 34 somites.

Growth in culture. Good growth in culture was assessed by strong heart beat and vigorous blood flow through cranial blood vessels. The somite number at the end of culture ranged from 38 to 43 somites, and the optimal total culture time was determined to be $\sim 14 \mathrm{hr}$. Embryos with $<38$ somites were not included in the analysis because of their short TPOC axons, in which only a few reached the VT/DT boundary. If Pax6 mutant embryos were cultured for $>16 \mathrm{hr}$, or to $>43$ somites, TPOC axons grew across the VT/DT boundary on both R-cadherin-targeted and control sides of the embryos, confounding the cadherin effects on axon outgrowth. This exuberant axon growth was likely an artifact of prolonged embryo culture conditions, because such growth was also observed in embryos that were not electroporated but simply cultured for 18 or more hours, or to $>43$ somites.
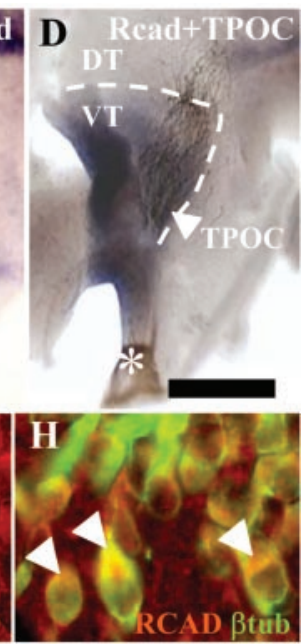

Electroporation targeting. Strong GFP fluorescence and coverage of the VT/DT region of the TPOC pathway were criteria used to screen embryos before axon analysis. Some embryos had GFP labeling that was restricted to regions dorsal to the TPOC pathway. These R-cadherin electroporations did not rescue TPOC axon growth (data not shown). Some embryos had weak or no GFP labeling, or GFP only outside of the TPOC pathway, and were not analyzed.

Tracing of axons. The initial DiI labeling was not symmetrical on some embryos, in which case an additional application of DiI was usually sufficient for equal labeling of the two sides of the embryo.

\section{Results}

$\mathrm{R}$-Cadherin and Pax6 are expressed in the pathway of the TPOC longitudinal pioneer axons

The TPOC axons pioneer a simple longitudinal tract through the forebrain (Fig. $1 A)$. The source of the axons is a cluster of neurons located at the base of the optic stalk, in the presumptive optic chiasm (Easter et al., 1993; Mason and Sretavan, 1997). Beginning on embryonic day 9.5, the axons project through the forebrain, first forming a narrow axon bundle that passes the ventral side of the optic stalk (Fig. 1A). Next, the axons fan out as they cross through the presumptive VT. By E10.5, the axons funnel together to cross into the DT and continue longitudinally into midbrain. Thus, the TPOC pioneers establish the main longitudinal tract through the forebrain, later followed by multitudes of descending and ascending followers. The stereotypical pattern of TPOC axons implies that they encounter specific cues along their pathway that direct their growth.

We showed previously that TPOC axons project specifically through the Pax6 ${ }^{+}$portion of the VT, whereas the TPOC neurons themselves do not express Pax6 (Mastick et al., 1997). Previous reports of R-cadherin expression patterns in older embryos identified high expression levels in ventral thalamus, as well as overlap with Pax6 (Matsunami and Takeichi, 1995; Stoykova et al., 1997). These observations suggested that R-cadherin expression likely overlaps the TPOC pathway, at least in the presumptive ventral thalamus. To test this idea, first, the mRNA expression patterns of Pax6 and R-cadherin were visualized by whole-mount in situ hybridization of embryos during the E9.5-E10.5 period of TPOC axon migration (Fig. $1 B, C$ ). On E10.5, R-cadherin in situ hybridization intensely labeled restricted regions of the alar forebrain, with the main labeled region consisting of VT and adjacent tissue extending both anteriorly through presumptive hypothalamus to include the optic stalk and dorsally into the cerebral vesicle. Expression of R-cadherin was present on E9.5 (data not shown), in the same pattern as on E10.5, showing that R-cadherin is present early, preceding the initiation of TPOC axon projections. We verified that, during the time of pioneer axon growth, Pax6 and R-cadherin extensively overlapped in VT, sharing boundaries of expression (Fig. $1 B, C$ ).

To directly compare the axon and R-cadherin patterns, the trajectory of the TPOC axons was correlated to R-cadherin through a double-labeling procedure (Fig. 1D). The TPOC axons were labeled anterogradely with the fluorescent lipophilic dye DiI, which was then photoconverted in the presence of DAB to a 
brown color, followed by whole-mount in situ hybridization for R-cadherin. These labels show that, for much of their trajectory through the forebrain, TPOC axons traverse R-cadherinexpressing tissue. Examination of double-labeled embryos showed that TPOC axons project specifically through the $\mathrm{Rcad}^{+}$ region of VT, closely respecting the ventral boundary of R-cadherin expression, before crossing into the DT (Fig. 1D). As the TPOC axons projected past the optic stalk early in the pathway, the axons entered the main R-cadherin expression domain, with the TPOC fully confined within this $\mathrm{R}-\mathrm{cad}^{+}$region as they passed through VT. The spatial correlation was close, although not complete. In particular, there was a very close correspondence between the ventral boundary of the R-cadherin expression pattern and the trajectory of the ventralmost TPOC axons. In addition, the VT/DT boundary (zona limitans interthalamica) was both an R-cadherin expression boundary and the place at which TPOC axons funnel together to cross the boundary. Interestingly, although much of the alar region of the ventral forebrain, including the cerebral vesicle, is $\mathrm{R}-\mathrm{cad}^{+}, \mathrm{TPOC}$ axons did not enter these areas.

The TPOC axons transit the VT within the pial (outermost) layer of the neuroepithelial wall, which consists of neuroepithelial end feet (Mastick et al., 1997). We examined sections through the VT to identify R-cadherin protein localization using R-cadherin antibody labeling. Labeling was concentrated in the pial layer (Fig. 1E,F). By comparing the R-cadherin pattern with Pax6, the pial layer of R-cadherin appears as a non-nuclear layer superficial to the Pax $6^{+}$neurons, consistent with the position of the neuroepithelial end feet. Together, these observations indicate that the local environment of the TPOC axonal growth cones is a specific subpial layer containing high levels of R-cadherin protein, suggesting a potential influence of R-cadherin on TPOC axon growth.

\section{The TPOC neurons express R-cadherin protein}

Cadherins require homophilic binding. Therefore, if substrate $\mathrm{R}$-cadherin directs the TPOC neurons, then the TPOC axons themselves should also express R-cadherin. Because of the high levels of R-cadherin expression present in the VT, it was not possible to directly verify $\mathrm{R}$-cadherin protein localization in the distal extensions of the TPOC axons. Therefore, to determine whether TPOC neurons express R-cadherin, R-cadherin antibody was used to label sections at the anterior base of the optic stalk. This region, the presumptive optic chiasm, contains the TPOC neuron cell bodies. On E10.5, the neuronal cell bodies in this region are the main source of the TPOC, based on observations that retrograde tracer applied to the TPOC axons labels this cluster of neuronal cell bodies and that anterograde tracing from these neuron cell bodies labels axon projections only into the TPOC (Mastick and Easter, 1996). Here, labeling of sections through the TPOC neuron cell bodies, revealed by anti- $\beta$ IIItubulin labeling, showed R-cadherin antibody labeling within the cell bodies and initial axon segments of the TPOC neurons (Fig. $1 G, H)$. This axonal expression of $\mathrm{R}$-cadherin protein in the TPOC axons could promote homophilic interactions with substrate R-cadherin in the VT.

R-cadherin expression is lost from the TPOC pathway of Pax6 mutant embryos, correlating with TPOC axon guidance errors

If R-cadherin is the Pax6-directed cue for TPOC axons in the VT, then its expression in E10.5 Pax6 $6^{-1-}$ embryos should be altered at the point at which axon errors occur. To examine R-cadherin
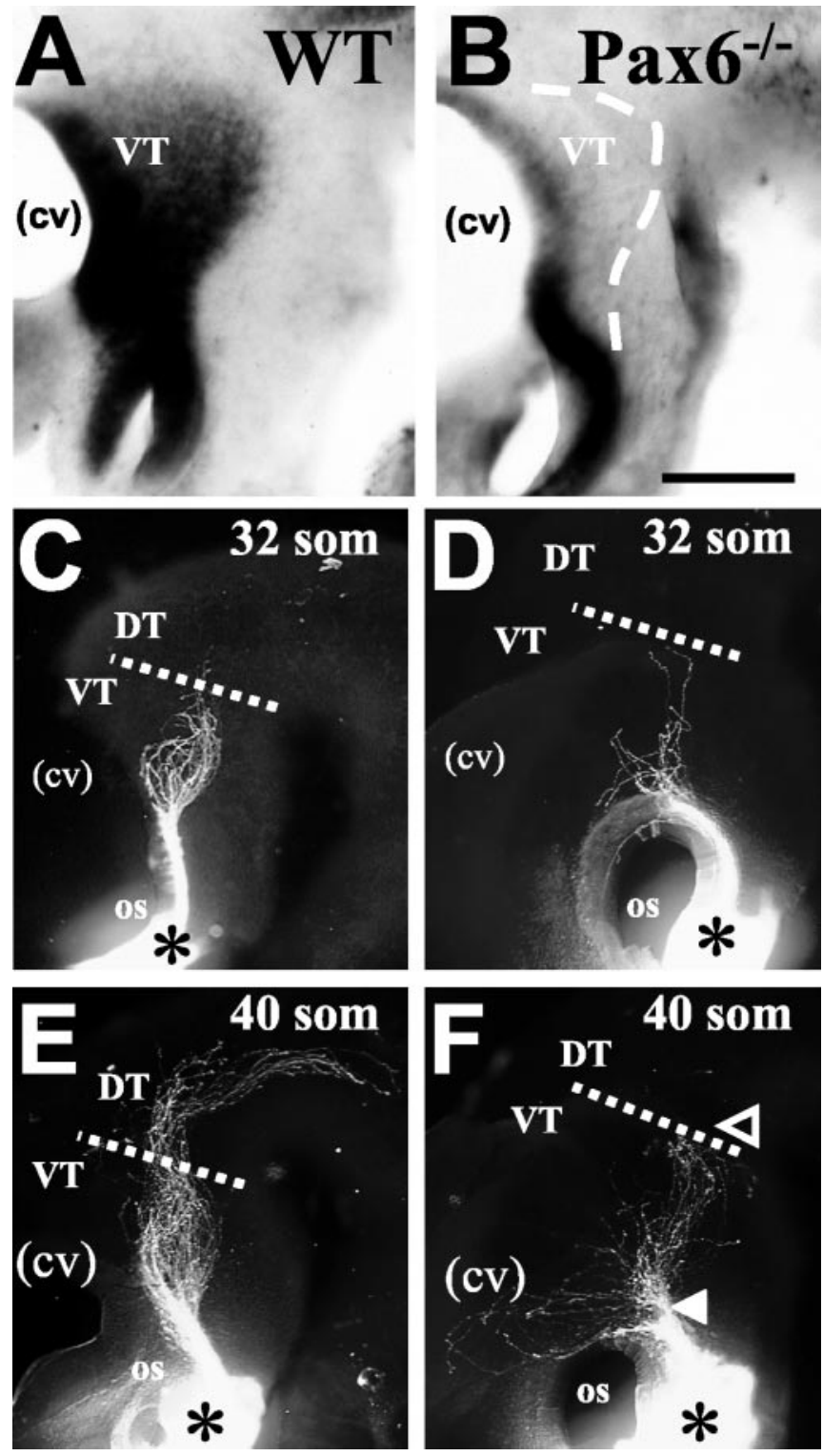

Figure 2. Loss of R-cadherin in Pax6 ${ }^{-1-}$ embryos correlates with axon errors. $A, B$ R-Cadherin in situ hybridization on E10.5, comparing wild-type (WT) and Pax6 ${ }^{-1-}$ embryos. Note selective loss of R-cadherin from VT in mutant $(B)$, although expression remains in optic stalk (os) and in cerebral vesicle (cv) (removed to reveal VT). C $-F$, Tracing of TPOC axons (asterisk, label site), comparing WT $(C, E)$ and mutant $(D, F)$ at two stages. Dashed line indicates the VT/DT boundary, which can be recognized by an external groove in both wild-type and Pax6 mutant embryos. C, D, Thirty-two somite (som) stage (early E10.5). In wild type, the leading axons approach the VT/DT boundary; in the mutant, axons are shorter and make loops in VT.E, $F$, Forty somite stage (E11). In wild type, more axons project along the pathway, crossing through DT and into midbrain. In mutant embryos $(F)$, a large proportion of axons stop (filled arrowhead) instead of growing into VT. Axon errors include projections into cerebral vesicle (cv) and dorsal turns at the VT/DT boundary instead of crossing (open arrowhead). The axon patterns shown are typical of $>100$ embryos that were labeled. Scale bar (in B): $A-F, 200 \mu \mathrm{m}$.

expression during the period of pioneer axon outgrowth in Pax6 mutant embryos, R-cadherin patterns were examined by in situ hybridization on wild-type and Pax6 mutant embryos on E10.5 (Fig. 2). In Pax6 mutants, the R-cadherin pattern was selectively altered (Fig. 2 B). R-Cadherin was specifically lost from VT. In contrast, R-cadherin expression was maintained at normal levels and patterns at the base of the optic stalk. Intense labeling was also maintained in the cerebral vesicle (data not shown). Thus, $\mathrm{R}$-cadherin expression appears to be regulated in a region- 
specific manner, with R-cadherin in the ventral thalamus strictly dependent on Pax6 for expression, whereas other neighboring regions such as optic stalk and cerebral vesicle retain $\mathrm{R}$-cadherin independent of Pax6 expression.

The change in R-cadherin expression in Pax6 mutants strongly correlates with alterations in TPOC axon navigation (Fig. 2C-F). TPOC axons made early and severe pathfinding errors in Pax6 mutant embryos (Fig. $2 D, F$ ) when compared with wild-type embryos (Fig. 2C,E). The earliest errors occurred where the axons would normally first encounter the Pax $6^{+} \mathrm{Rcad}^{+}$region (Fig. 2D). At this point in early embryos, axons could be seen to fan out in several directions. This choice point bordered the normal pathway that was now $\mathrm{Rcad}^{-}$, as well as the base of the optic stalk and the cerebral vesicle, which were $\mathrm{Rcad}^{+}$. Thus, as soon as the TPOC axons encountered the abnormal $\mathrm{Rcad}^{-}$pathway, they made immediate errors.

In older embryos (40 somites), the number and severity of axon errors continued to increase. These errors included a large number of axons that terminated in the vicinity of the aberrant choice point, suggesting that the growth of most axons was stalled. Some of the axons that projected past the choice point grew in aberrant directions never seen in wild-type embryos, including continuing around the base of the optic stalk, as well as long projections into the cerebral vesicle. Growth into either the optic stalk or cerebral vesicle led the axons into $\mathrm{Rcad}^{+}$regions (Fig. $2 \mathrm{~B}$ ). Axons that projected into $\mathrm{Rcad}^{+}$regions tended to be long and without loops. Some axons did project in the proper VT pathway as expected in wild type, but these were shorter than in wild type and tended to form loops. The VT/DT boundary was less prominent in Pax6 mutants than in wild type, but a distinct neuromere-like morphological constriction was quite visible (Mastick et al., 1997). Significantly, this boundary was reached by some axons, but the axons turned to project dorsally along the boundary instead of crossing into DT. Thus, TPOC axons that grew in contact with $\mathrm{R}$-cadherin had long straight projections, although projecting through inappropriate regions. In contrast, those TPOC axons that did project in the appropriate direction but encountered the $\mathrm{Rcad}^{-}$pathway had trajectories that suggested that the $\mathrm{Rcad}^{-}$pathway was not optimal for growth. We conclude that, in the absence of Pax6, TPOC axons make pathfinding errors that are closely correlated with selective alterations in the pattern of R-cadherin. The observations of inhibited growth along the pathway that now lacks R-cadherin expression, coupled with exuberant growth into aberrant but $\mathrm{Rcad}^{+}$regions, are consistent with $\mathrm{R}$-cadherin functioning as a growthpromoting cue for TPOC axons.

\section{R-cadherin promotes TPOC axon outgrowth}

To directly test how TPOC axons respond to a defined $\mathrm{R}$-cadherin substrate, an explant assay was used in which TPOC axons were grown in vitro on cellular substrates either with or without R-cadherin (Fig. 3). Small pieces of forebrain tissue containing the TPOC neuron cell bodies were dissected from wildtype mouse embryos, placed on top of monolayer cultures of fibroblasts, and incubated for $24 \mathrm{hr}$. When cultured on fibroblasts engineered to express R-cadherin, TPOC axons grew significantly longer than when cultured on cells lacking cadherin expression (Fig. $3 A, B, D, E$ ). In the absence of R-cadherin, TPOC axons grew only short distances from the explants, with strong fasciculation, suggesting a bias toward growing on neighboring $\mathrm{Rcad}^{+}$axons rather than on the $\mathrm{Rcad}^{-}$substrate (Fig. 3B). In addition, explants on $\mathrm{Rcad}^{-}$substrates had high numbers of axons within the explant itself (data not shown), suggesting that
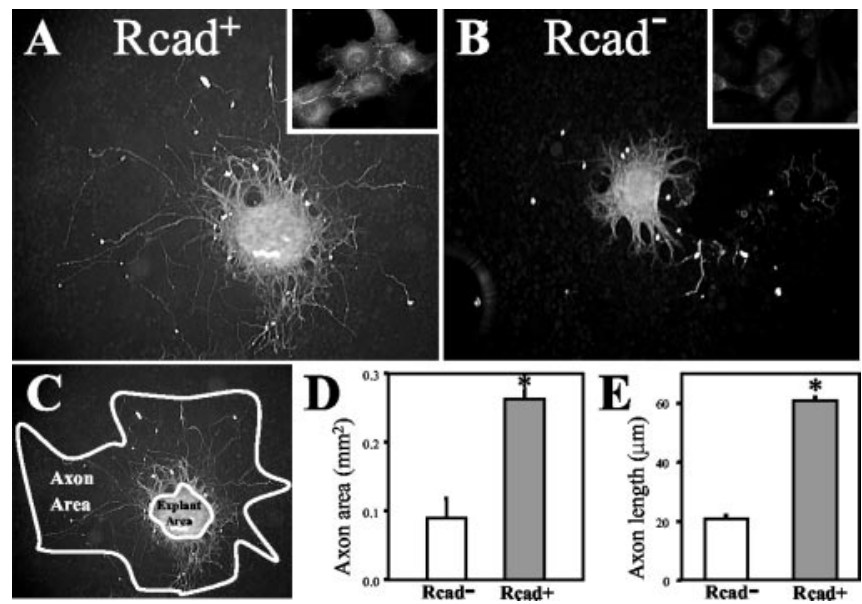

Figure 3. R-Cadherin substrate promotes TPOC axon outgrowth in vitro. Tissue containing TPOC cell bodies was dissected from mouse embryos and placed onto monolayers of fibroblasts, either stably transfected with R-cadherin $(A)$ or control cells lacking any cadherin expression $(B)$. Insets in $A$ and $B$ are high-magnification images of cell lines labeled with R-cadherin antibody. After $24 \mathrm{hr}$ of culture, the explants were fixed and labeled with neuron-specific $\beta$-tubulin antibody to visualize the axons for quantification. The results shown are typical of at least three independent experiments. $C$, The extent of axon outgrowth on the two substrates was estimated by comparing the area within the perimeter of the longest axons. $D$, Graph comparing the axon growth area (mean \pm SEM) between the control and R-cadherin substrates, with a significant increase on the R-cadherin substrate ( $t$ test; $p<0.001$; explant numbers: $\operatorname{Rcad}^{+}, n=10 ; \mathrm{Rcad}^{-}, n=9$; the sum of two independent experiments for each condition). E, Graph comparing the length (mean \pm SEM) of the axons (longest 14-20 per explant) between the control and R-cadherin substrates ( $p \ll 0.001$; explant numbers: Rcad $\left.^{+}, n=19 ; \operatorname{Rcad}^{-}, n=9\right)$.

many axons failed to exit, or grew back into, explants placed on $\mathrm{Rcad}^{-}$substrates. Thus, when presented with an $\mathrm{Rcad}^{+}$substrate in vitro, the outgrowth of TPOC axons was promoted. The ability of the axons to respond to $\mathrm{Rcad}^{+}$substrate suggests that the defects in TPOC projection patterns in $\mathrm{Pax}^{-1-}$ mouse embryos is attributable to the loss of R-cadherin from the TPOC pathway.

\section{$\mathrm{R}$-cadherin expression is sufficient to rescue TPOC axon growth in Pax6 mutant embryos}

The loss of Pax6 could result in multiple molecular defects in ventral thalamic development, in addition to loss of R-cadherin. If loss of R-cadherin expression makes a critical contribution to TPOC guidance errors in Pax6 mutant embryos, then selective restoration of R-cadherin expression in these embryos should rescue TPOC axon growth, for example, through the VT and into the DT. To restore R-cadherin expression to Pax6 mutant embryos, an R-cadherin expression plasmid (Inoue et al., 2001) was electroporated into the forebrain of intact E10.5 Pax6 mutant embryos, followed by whole mouse embryo culture for $14 \mathrm{hr}$ (Fig. 4) (Osumi and Inoue, 2001; Swartz et al., 2001). To affect TPOC axons at the beginning of their migration, only embryos at early E10.5 (31-34 somites) were electroporated. Electroporated cells covered the TPOC pathway, as visualized by coelectroporation of a GFP plasmid (Fig. 4E). Because of the polarity of the electrode chamber, electroporation of the plasmid DNA was unilateral, thereby leaving the other side of the embryo as an untransfected internal control TPOC pathway that lacked R-cadherin expression. There was significant axon growth on the $\mathrm{Rcad}^{+}$side, with many axons growing into VT and crossing into DT (Fig. 4C,D). On the control side, fewer axons grew into VT, and only a small number crossed into DT (Fig. $4 A, B$ ). The TPOC axons on the $\mathrm{Rcad}^{+}$side were substantially longer than on the control side, 

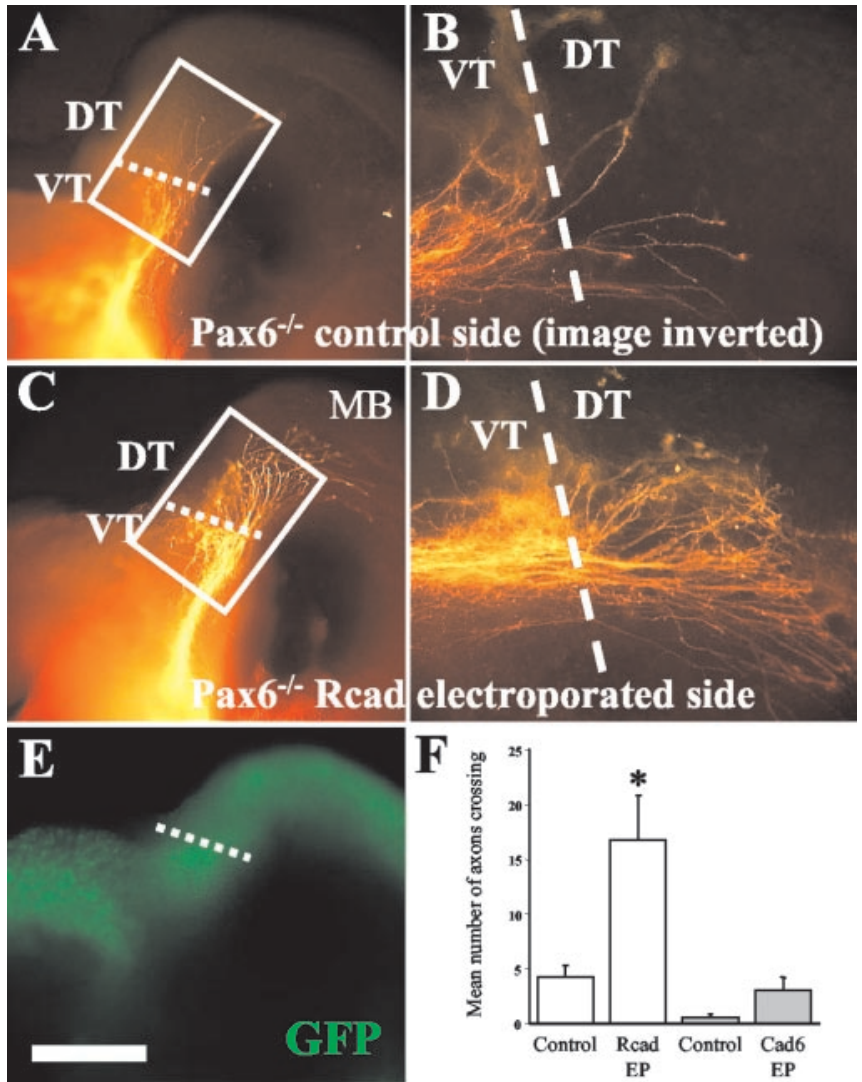

Figure 4. Rescue of axon growth in Pax6 $6^{-1-}$ embryos by electroporation of R-cadherin $\mathrm{Pax}^{-1-}$ embryos were electroporated with a mixture of R-cadherin and GFP expression plasmids, followed by whole embryo culture. $A$, Control (anode) side of an electroporated Pax6 ${ }^{-1-}$ embryo. Axon projections are typical of Pax6 mutants, with some axons growing to the VT/DT boundary but few crossing. Mirror image is shown to facilitate comparison with electroporated side. $B$, High-magnification view of axons on control side. $C-E$, Other side of same Pax6 $6^{-1-}$ embryo, electroporated with R-cadherin and GFP, with GFP pattern shown in E. C, Targeted (cathode) side. Axons project past the VT/DT boundary, with the longest axons reaching the midbrain (MB). D, High-magnification view of R-cadherin electroporated side, showing increased numbers of axons growing to and across boundary. F, Quantification of boundary crossing phenotype in R-cadherin and cadherin-6 electroporated Pax6 ${ }^{-1-}$ embryos. R-Cadherin electroporation led to a significantly increased mean number of axons crossing compared with the control side of each embryo ( $p<0.01$; paired $t$ test; $n=10$ summed from 7 independent experiments). In contrast, cadherin-6 electroporations (Cad6 EP) resulted in significantly fewer axons crossing than for R-cadherin electroporations $(\operatorname{Rcad} E P)(p<0.01 ; t$ test; $n=4$ summed from 2 independent experiments). Scale bar (in $E): A, C, E, 200 \mu \mathrm{m} ; B, D, 100 \mu \mathrm{m}$.

with some axons extending into the midbrain. Quantitative analysis showed that many more axons crossed into DT on the $\mathrm{Rcad}^{+}$ side than on the control side (Fig. $4 F$ ). The R-cadherin-mediated rescue was apparently a local effect, because rescued TPOC axon projections grew specifically through $\mathrm{GPF}^{+}$transfected patches of VT/DT and failed to be rescued by mistargeted patches, such as extreme dorsal DT (data not shown). To control for cadherin specificity, cadherin-6, which fails to bind to R-cadherin (Inoue et al., 2001), was instead electroporated into Pax6 mutant embryos. There was no significant rescue in embryos electroporated with cadherin-6 (Fig. 4F), consistent with a requirement for $\mathrm{R}$-cadherin-mediated homophilic interactions between TPOC axons and substrate cells. The enhanced axon growth after $\mathrm{R}$-cadherin electroporation indicates that $\mathrm{R}$-cadherin is sufficient to restore TPOC axon growth along the pathway and to stimulate axon crossing from ventral thalamus to dorsal thalamus.

\section{Discussion}

This study has generated several independent lines of evidence supporting a role for R-cadherin as a guidance cue for the TPOC pioneer axons in the embryonic mouse brain. R-Cadherin is present at the right time and place to influence the axons, and $\mathrm{R}$-cadherin expression is altered in Pax6 mutant embryos, closely correlating with specific axon errors. Both in vitro and in vivo experiments provided evidence that $\mathrm{R}$-cadherin can promote the growth of the pioneer axons. Together, these results suggest that $\mathrm{R}$-cadherin provides a growth-promoting substrate in the forebrain that guides the TPOC axons through a segment of their pathway.

This hypothesis explains several aspects of TPOC axon navigation in wild-type embryos, as well as the disrupted axon patterns in Pax6 mutants. The initial segment of axonal growth consists of the initial axon projections from the TPOC neuronal cell bodies past the optic stalk, projections that are normal in Pax6 mutants (Fig. 2). After rounding the optic stalk, in contrast, axons in wild-type embryos encounter and fan out over a large region of $\mathrm{Rcad}^{+}$cells. Because R-cadherin substrate can stimulate TPOC axon outgrowth in vitro, we propose that $\mathrm{Rcad}^{+}$cell surfaces serve to promote the growth of the TPOC axons through the VT segment of their pathway.

Pax6 mutant embryos provide a challenge to the TPOC axons in the form of an altered R-cadherin pattern. Strikingly, three aspects of TPOC axon projections are abnormal in Pax6 mutants (Mastick et al., 1997). First, after encountering the $\mathrm{Rcad}^{-}$pathway, many TPOC axons apparently stall and fail to project any farther (Fig. 2). This behavior is consistent with the loss of a growth-promoting substrate. Those axons that do project into the $\mathrm{Rcad}^{-}$pathway tend to form loops and are much shorter than in wild type. Second, after the stalling point, a subset of axons diverge from the normal pathway to grow long distances into the cerebral vesicle and the optic stalk. Strikingly, these aberrant projections are made into regions that retain high levels of R-cadherin expression. These observations suggest that, when faced with an $\mathrm{R}_{-} \mathrm{cad}^{-}$pathway, TPOC axon growth can be strongly promoted into aberrant regions that are $\mathrm{Rcad}^{+}$, behavior that provides additional evidence of the growth-promoting properties of R-cadherin.

The third abnormal aspect of pioneer axon navigation in Pax6 mutants is the failure of TPOC axons to cross from VT into DT. The simplest explanation is that DT, or the VT/DT boundary itself, contains a Pax6-dependent cue required for TPOC axon entry into DT. This Pax6-dependent cue could be cellular and/or molecular, but because DT does not express R-cadherin, the missing cue must be R-cadherin independent. Several Pax6 functions in DT development have been identified, including proliferative defects and altered regulatory gene expression (Stoykova et al., 1996; Grindley et al., 1997; Warren and Price, 1997; Pratt et al., 2000). More specifically, for axon guidance, axons that ascend through DT make errors in Pax6 mutants, including the tyrosine hydroxylase $^{+}$nigrostriatal and mammillothalamic axons (Valverde et al., 2000; Vitalis et al., 2000). In addition, the thalamocortical axons, which originate in DT, make errors in Pax6 mutants (Kawano et al., 1999; Hevner et al., 2002; Jones et al., 2002; Pratt et al., 2002). These observations suggest that multiple populations of axons depend on Pax6 to transit through DT and/or VT.

The model for guidance of TPOC axons by R-cadherin has an attractive simplicity, but a number of observations imply, not surprisingly, that TPOC axons have additional influences on 
their guidance. It is likely that many aspects of TPOC guidance are independent of R-cadherin or Pax6. Because much genetic patterning of the forebrain as well as neuronal differentiation patterns remain primarily unchanged in Pax6 mutant embryos (Stoykova et al., 1996; Grindley et al., 1997; Mastick et al., 1997; Warren and Price, 1997; Vitalis et al., 2000; Mastick and Andrews, 2001), many molecular or cellular cues may remain for the TPOC axons. First, R-cadherin patterns are not sufficient to fully explain the normal pattern of TPOC axon projections in wildtype embryos. The clearest case is that the pathway chosen by the TPOC axons is restricted to only the ventralmost subregion of the R-cadherin expression in VT (Fig. 1). Thus, R-cadherin expression is not normally sufficient to promote TPOC axon outgrowth into any brain region, because, although R-cadherin expression extends dorsally from the midpoint of the diencephalon up to include much of the cerebral vesicle, the TPOC axons are ventrally restricted. Because the axons apparently have full access to the contiguous $\mathrm{Rcad}^{+}$region, additional positive cues may be present to keep the axons within the pathway, or inhibitory cues may be present to restrict the axons from the dorsal $\mathrm{Rcad}^{+}$tissue, or a combination of positive and negative cues. Additionally, the factors that guide the directionality of TPOC axon growth remain unclear, because the R-cadherin pattern has no discernable concentration gradient, on either mRNA or protein levels. One possibility to consider is that directionality may derive from an initial polarity of the TPOC neurons and that directional growth could be maintained by the strong tendency of axons to grow straight in the absence of other cues (Katz, 1985).

The ability of R-cadherin electroporation to rescue TPOC axon growth in Pax6 mutant embryos provided an in vivo assay demonstrating that TPOC axons can respond to R-cadherin substrates. Furthermore, this assay allowed a test of the relative importance of the lost expression of R-cadherin in Pax6 mutants, through the replacement of R-cadherin uniquely in the context of Pax6 mutant tissue. This electroporation strategy allowed the selective replacement of R-cadherin expression into the TPOC pathway of Pax6 mutant embryos, just before the axons grew through this region, followed by culture for $14 \mathrm{hr}$ to allow the axons to respond to their new molecular environment (Fig. 4). Because R-cadherin alone can rescue specific axon errors in Pax6 mutants, this result implicates R-cadherin as a key mediator of Pax6 action in axon guidance. R-Cadherin electroporation had the dual effect of increasing the number of axons that grow through VT and subsequently rescuing the ability of TPOC axons to cross into DT. Thus, although other molecular and cellular defects are likely present in the VT of Pax6 mutant embryos, $\mathrm{R}$-cadherin expression alone was sufficient to rescue axon growth. R-Cadherin rescue of axon growth could involve either specific homophilic molecular interactions mediating axon-substrate interactions or a more general effect, such as modifying neuroepithelial tissue organization. To distinguish between these two possibilities, electroporation was performed with cadherin-6, a cadherin that does not interact with R-cadherin (Inoue et al., 2001). Cadherin-6 failed to affect TPOC axon growth, suggesting that homophilic R-cadherin interactions were required for TPOC axon guidance. One potential limitation of the electroporation technique is the broad pattern of expression created. For example, the R-cadherin rescue created a broad swath of R-cadherin in the forebrain, also including DT, which does not normally express R-cadherin. More generally, this electroporation technique is a potentially powerful strategy for testing gene function in embryonic development of the brain and other tissues (Osumi and Inoue, 2001; Swartz et al., 2001).
The specific loss of VT R-cadherin expression in Pax6 mutants indicates that Pax6 is an important regulator of R-cadherin expression. This observation confirms a previous study done on older Pax6 mutant mouse embryos (Stoykova et al., 1997). Pax6 regulation of $\mathrm{R}$-cadherin appears to be general for vertebrates, because zebrafish R-cadherin expression is downregulated after decreased Pax6 expression and upregulated after increased Pax6 expression (Liu et al., 2001). The R-cadherin promoter has not been characterized, leaving open the question of whether Pax6 regulation of R-cadherin is direct or indirect. Pax6 and $\mathrm{R}$-cadherin expression only partially overlap, so other factors must contribute to R-cadherin regulation.

Cadherins evolved with the advent of multicellular animals, with the primary function of creating distinct, self-adhering populations of cells. This primary function of cadherins reaches its epitome in the vertebrate brain, in which complex cadherin expression patterns subdivide and organize diverse cell populations (Redies, 2000; Inoue et al., 2001; McCarthy et al., 2001). Our results suggest that this patchwork of cadherin-expressing cell populations has a second, emergent function of providing a patterned adhesive substrate for axons in transit through the brain.

\section{References}

Anderson RB, Key B (1999) Novel guidance cues during neuronal pathfinding in the early scaffold of axon tracts in the rostral brain. Development 126:1859-1868.

Chitnis AB, Kuwada JY (1991) Elimination of a brain tract increases errors in pathfinding by follower growth cones in the zebrafish embryo. Neuron 7:277-285.

Dickson BJ (2002) Molecular mechanisms of axon guidance. Science 298:1959-1964.

Easter Jr SS, Ross LS, Frankfurter A (1993) Initial tract formation in the mouse brain. J Neurosci 13:285-299.

Grindley JC, Hargett LK, Hill RE, Ross A, Hogan BL (1997) Disruption of PAX6 function in mice homozygous for the Pax6Sey-1Neu mutation produces abnormalities in the early development and regionalization of the diencephalon. Mech Dev 64:111-126.

Harris WA (1989) Local positional cues in the neuroepithelium guide retinal axons in embryonic Xenopus brain. Nature 339:218-221.

Hevner RF, Miyashita-Lin E, Rubenstein JL (2002) Cortical and thalamic axon pathfinding defects in Tbr1, Gbx2, and Pax6 mutant mice: evidence that cortical and thalamic axons interact and guide each other. J Comp Neurol 447:8-17.

Hibbard E (1965) Orientation and directed growth of Mauthners cell axons form duplicated vestibular nerve roots. Exp Neurol 13:289-301.

Inoue T, Tanaka T, Takeichi M, Chisaka O, Nakamura S, Osumi N (2001) Role of cadherins in maintaining the compartment boundary between the cortex and striatum during development. Development 128:561-569.

Jones L, Lopez-Bendito G, Gruss P, Stoykova A, Molnar Z (2002) Pax6 is required for the normal development of the forebrain axonal connections. Development 129:5041-5052.

Katz MJ (1985) How straight do axons grow? J Neurosci 5:589-595.

Kawano H, Fukuda T, Kubo K, Horie M, Uyemura K, Takeuchi K, Osumi N, Eto K, Kawamura K (1999) Pax-6 is required for thalamocortical pathway formation in fetal rats. J Comp Neurol 408:147-160.

Kidd T, Brose K, Mitchell KJ, Fetter RD, Tessier-Lavigne M, Goodman CS, Tear G (1998) Roundabout controls axon crossing of the CNS midline and defines a novel subfamily of evolutionarily conserved guidance receptors. Cell 92:205-215.

Liu Q, Sanborn KL, Cobb N, Raymond PA, Marrs JA (1999) R-cadherin expression in the developing and adult zebrafish visual system. J Comp Neurol 410:303-319.

Liu Q, Marrs JA, Chuang JC, Raymond PA (2001) Cadherin-4 expression in the zebrafish central nervous system and regulation by ventral midline signaling. Brain Res Dev Brain Res 131:17-29.

Mason CA, Sretavan DW (1997) Glia, neurons, and axon pathfinding during optic chiasm development. Curr Opin Neurobiol 7:647-653.

Mastick GS, Andrews GL (2001) Pax6 regulates the identity of embryonic diencephalic neurons. Mol Cell Neurosci 17:190-207. 
Mastick GS, Easter Jr SS (1996) Initial organization of neurons and tracts in the embryonic mouse fore- and midbrain. Dev Biol 173:79-94.

Mastick GS, Davis NM, Andrew GL, Easter Jr SS (1997) Pax-6 functions in boundary formation and axon guidance in the embryonic mouse forebrain. Development 124:1985-1997.

Matsunaga M, Hatta K, Nagafuchi A, Takeichi M (1988) Guidance of optic nerve fibres by N-cadherin adhesion molecules. Nature 334:62-64.

Matsunami H, Takeichi M (1995) Fetal brain subdivisions defined by Rand E-cadherin expressions: evidence for the role of cadherin activity in region-specific, cell-cell adhesion. Dev Biol 172:466-478.

Matsunami H, Miyatani S, Inoue T, Copeland NG, Gilbert DJ, Jenkins NA, Takeichi M (1993) Cell binding specificity of mouse R-cadherin and chromosomal mapping of the gene. J Cell Sci 106:401-409.

McCarthy M, Na E, Neyt C, Langston A, Fishell G (2001) Calciumdependent adhesion is necessary for the maintenance of prosomeres. Dev Biol 233:80-94.

Nakamura S, Ito Y, Shirasaki R, Murakami F (2000) Local directional cues control growth polarity of dopaminergic axons along the rostrocaudal axis. J Neurosci 20:4112-4119.

Osumi N, Inoue T (2001) Gene transfer into cultured mammalian embryos by electroporation. Methods 24:35-42.

Parr BA, Shea MJ, Vassileva G, McMahon AP (1993) Mouse Wnt genes exhibit discrete domains of expression in the early embryonic CNS and limb buds. Development 119:247-261.

Pratt T, Vitalis T, Warren N, Edgar JM, Mason JO, Price DJ (2000) A role for Pax6 in the normal development of dorsal thalamus and its cortical connections. Development 127:5167-5178.

Pratt T, Quinn JC, Simpson TI, West JD, Mason JO, Price DJ (2002) Disruption of early events in thalamocortical tract formation in mice lacking the transcription factors Pax6 or Foxg1. J Neurosci 22:8523-8531.

Redies C (2000) Cadherins in the central nervous system. Prog Neurobiol 61:611-648.

Redies C, Engelhart K, Takeichi M (1993) Differential expression of N- and
R-cadherin in functional neuronal systems and other structures of the developing chicken brain. J Comp Neurol 333:398-416.

Riehl R, Johnson K, Bradley R, Grunwald GB, Cornel E, Lilienbaum A, Holt $\mathrm{CE}$ (1996) Cadherin function is required for axon outgrowth in retinal ganglion cells in vivo. Neuron 17:837-848.

Stone KE, Sakaguchi DS (1996) Perturbation of the developing Xenopus retinotectal projection following injections of antibodies against betal integrin receptors and N-cadherin. Dev Biol 80:297-310.

Stoykova A, Fritsch R, Walther C, Gruss P (1996) Forebrain patterning defects in Small eye mutant mice. Development 122:3453-3465.

Stoykova A, Gotz M, Gruss P, Price J (1997) Pax6-dependent regulation of adhesive patterning, R-cadherin expression and boundary formation in developing forebrain. Development 124:3765-3777.

Swartz M, Eberhart J, Mastick GS, Krull CE (2001) Sparking new frontiers: using in vivo electroporation for genetic manipulations. Dev Biol 233:13-21.

Treubert-Zimmermann U, Heyers D, Redies C (2002) Targeting axons to specific fiber tracts in vivo by altering cadherin expression. J Neurosci 22:7617-7626.

Valverde F, Garcia C, Lopez-Mascaraque L, De Carlos JA (2000) Development of the mammillothalamic tract in normal and Pax-6 mutant mice. J Comp Neurol 419:485-504.

Vitalis T, Cases O, Engelkamp D, Verney C, Price DJ (2000) Defects of tyrosine hydroxylase-immunoreactive neurons in the brains of mice lacking the transcription factor pax6. J Neurosci 20:6501-6516.

Walther C, Gruss P (1991) Pax-6, a murine paired box gene, is expressed in the developing CNS. Development 113:1435-1449.

Warren N, Price DJ (1997) Roles of Pax-6 in murine diencephalic development. Development 124:1573-1582.

Wilkinson DG (1992) Whole mount in situ hybridization of vertebrate embryos. In: In situ hybridization: a practical approach (Wilkinson DG, ed), pp 75-83. New York: Oxford UP. 